\title{
SnoN expression is differently regulated in microsatellite unstable compared with microsatellite stable colorectal cancers June A Chia ${ }^{1}$, Lisa A Simms ${ }^{1}$, Sarah-Jane Cozzi ${ }^{1}$, Joanne Young ${ }^{2}$, Jeremy R Jass ${ }^{3}$, Michael D Walsh ${ }^{2}$, Kevin J Spring ${ }^{1}$, Barbara A Leggett ${ }^{1}$ and Vicki LJ Whitehall*1
}

Address: ${ }^{1}$ The Conjoint Gastroenterology Laboratory, Royal Brisbane and Women's Hospital Foundation Clinical Research Centre and the Queensland Institute of Medical Research, Brisbane, 4029, Australia, ${ }^{2}$ The Molecular Cancer Epidemiology Laboratory, The Queensland Institute of Medical Research, Brisbane, 4029, Australia and ${ }^{3}$ The Department of Pathology, McGill University, Montreal, H3A 2B4, Canada

Email: June A Chia - June.Chia@qimr.edu.au; Lisa A Simms - Lisa.Simms@qimr.edu.au; Sarah-Jane Cozzi - Sarah-Jane.Cozzi@qimr.edu.au; Joanne Young - Joanne.Young@qimr.edu.au; Jeremy R Jass - jeremy.jass@mcgill.ca; Michael D Walsh - Michael.Walsh@qimr.edu.au; Kevin J Spring - Kevin.Spring@qimr.edu.au; Barbara A Leggett - Barbara_Leggett@health.qld.gov.au;

Vicki LJ Whitehall* - Vicki.Whitehall@qimr.edu.au

* Corresponding author

Published: 24 October 2006

BMC Cancer 2006, 6:252 doi:10.1 I86/147|-2407-6-252
Received: 29 June 2006

Accepted: 24 October 2006

This article is available from: http://www.biomedcentral.com//47/-2407/6/252

(c) 2006 Chia et al; licensee BioMed Central Ltd.

This is an Open Access article distributed under the terms of the Creative Commons Attribution License (http://creativecommons.org/licenses/by/2.0), which permits unrestricted use, distribution, and reproduction in any medium, provided the original work is properly cited.

\section{Abstract}

Background: $\mathrm{SnoN}$ is an important regulator of the transforming growth factor beta (TGF $\beta$ ) signalling pathway and has been shown to exhibit both tumour promotion and suppression activity.

Methods: To further explore the role of this complex molecule in colorectal tumorigenesis, we examined 52 paired normal and tumour colorectal specimens stratified by level of microsatellite instability; 18 with high-level microsatellite instability (MSI-H) and 34 microsatellite stable (MSS). SnoN transcript expression was quantitated by real-time PCR and analysed with respect to clinical indicators of prognosis.

Results: Within the MSI-H subgroup, SnoN was commonly either up-regulated $(6 / 18,33 \%)$ or down-regulated (7//8, 39\%). A significantly different distribution of SnoN expression was observed in MSS cancers compared with MSI-H $(P \leq 0.00 I)$. Whilst $17 / 34(50 \%)$ of MSS tumours demonstrated up-regulation, none showed down-regulated expression. Within the MSI-H subgroup, up-regulation was significantly correlated with lack of repeat tract mutation in the $T G F \beta R$ Il gene $(P \leq 0.025)$, suggesting that $S n o N$ is more frequently up-regulated in the presence of functional TGF $\beta$ signalling.

Conclusion: Together these data support the notion that SnoN has both oncogenic and tumour suppressive properties depending on other genetic changes within the tumour, and that the MSI-H pathway of colorectal tumorigenesis presents an excellent model for the study of these opposing functions. 


\section{Background}

SnoN (ski-related novel gene) is a member of the highly homologous Ski gene family [1], and can act as a repressor of the TGF- $\beta$ signalling pathway through either SnoN homodimerisation or heterodimerisation with Ski [1-6]. This repression is mediated through binding of SnoN to SMAD proteins, which are responsible for the propagation of signals initiated by TGF- $\beta$ ligand for the transcription of genes required for cell growth control and differentiation $[1,4,7,8]$. Conversely, SnoN has also been shown to suppress tumour growth both in a heterozygote mouse model of SnoN inactivation [9] and in human cells [10]. These opposing functions have led to difficulty in interpreting the complex roles of SnoN in human malignancies.

Both SnoN and Ski have been implicated in the development of human cancers including colon [11], breast [12], melanoma [3] and oesophageal cancers [13]. Expression of $\mathrm{SnoN}$ has not previously been examined in colorectal cancer (CRC), although a study of gene copy number status showed that the SnoN locus was partially or completely deleted in 55\% of CRCs and amplified in 15\% [11]. Interestingly, increased copy number of $S k i$ was found to be a negative prognostic indicator whilst no correlation with patient outcome was observed for SnoN. Since regulation of TGF $\beta$ signalling by SnoN involves a combination of proteolytic degradation and transcriptional up-regulation in addition to changes in copy number, we suggest that quantification of SnoN expression may be a more informative way to assess its role in colorectal tumour development.

Colorectal tumours stratified by level of microsatellite instability represent two principal pathways of sporadic colorectal tumorigenesis. The majority of sporadic CRCs are microsatellite stable (MSS) and aneuploid. These have been well studied but are understood to be heterogeneous at the molecular level [14]. Cancers displaying a high level of microsatellite instability (MSI-H) comprise approximately $15 \%$ of all sporadic CRCs, are diploid and generally have a favourable prognosis [15]. Whilst the major mechanisms for gene inactivation in MSS cancers are mutation and deletion, MSI-H tumours progress predominantly due to repeat-tract mutation and aberrant promoter hypermethylation of important target tumour suppressor genes [16].

We have examined the frequency of altered SnoN expression in a series of CRCs stratified by MSI-status. Based on our findings we propose that MSI-H cancers present an excellent model for studying the dual roles of $\mathrm{SnoN}$ in both tumour suppression and promotion.

\section{Methods \\ Patient specimens}

Fifty-two paired normal and tumour tissue specimens were obtained in a fresh state from colectomy specimens surgically resected at the Royal Brisbane and Women's Hospital between 1993 and 2005. The Human Research Ethics Committee of the Queensland Institute of Medical Research and the Royal Brisbane and Women's Hospital Research Ethics Committee approved this study and all patients provided written informed consent. The standard panel of markers (BAT25, BAT26, D5S346, D2S123 and D17S250) recommended by a National Cancer Institute workshop were used to determine the MSI-status of the cancers, as previously described [17]. Patients were classified as microsatellite instability high (MSI-H) if $>30 \%$ of markers showed instability. The remainder of patients were microsatellite stable (MSS). The patient cohort was enriched for MSI-H tumours, but was otherwise unselected. Patients with familial adenomatous polyposis (FAP) or hereditary non-polyposis colorectal cancer (HNPCC) were excluded from the study.

\section{Expression analysis}

Total RNA was extracted from tissue samples which had been snap frozen in liquid nitrogen then stored at $-80^{\circ} \mathrm{C}$ using the QIAGEN RNeasy Midi Kit. Total RNA ( $5 \mu \mathrm{g})$ was reverse transcribed using random hexamers and SuperScript III Reverse Transcriptase (Invitrogen, USA). Semiquantitative real-time polymerase chain reaction was performed using a Corbett Rotor-Gene 3000 machine (Corbett Research, Australia) with the following gene specific primers: Reference gene, $\beta$-ActinF: 5'-TCATGAAGTGTGACGTGGACATC-3'; $\beta$-ActinR: 5 '-CAGGAGGAGCAATGATCTTGATCT-3'; Gene Of Interest, SnoNF: 5'CACCCCAGCTACTACTTATAC-3', SnoNR: 5'TTGCCTCTGTCTTTGTGAGC-3') and Platinum SYBR Green qPCR Supermix-UDG (Invitrogen, USA). Each reaction contained $8 \mu \mathrm{L}$ of qPCR mix, 5 pmol of each forward and reverse primer and $4 \mu \mathrm{L}$ of diluted cDNA template. The following cycling conditions were applied: $50^{\circ} \mathrm{C}$ for 2 $\min , 95^{\circ} \mathrm{C}$ for $2 \mathrm{~min}$, followed by 45 cycles of $95^{\circ} \mathrm{C}$ for 15 sec, $59^{\circ} \mathrm{C}$ for $15 \mathrm{sec}, 72^{\circ} \mathrm{C}$ for $20 \mathrm{sec}, 76^{\circ} \mathrm{C}$ of $15 \mathrm{sec}$. Data for each cycle was acquired after the final cycling step. A melt ramp consisting of $1^{\circ} \mathrm{C}$ increments from $60^{\circ} \mathrm{C}$ to $99^{\circ} \mathrm{C}$ (30 sec first step, $1 \mathrm{sec}$ for subsequent steps) followed the 45 cycles. Each reaction was carried out in duplicate (patient samples) or triplicate (calibrator). The relative expression of SnoN to $\beta$-Actin was calculated using the method previously described by Pfaffl [18]. qPCR results from each set of reaction replicates varied no more than 5\% from the mean. Normalised SnoN expression values $(\mathrm{R})$ for all tumours were divided by the R value for their matched normal mucosa to indicate fold up-regulation, the inverse gave a whole number to indicate fold down-regulation. Data was analysed based on empirical 
fold differences (student's T-test) as well as number of tumours showing greater than 2 fold up- or down-regulation (Pearson's chi square). The cut-off of 2-fold was arbitrarily chosen as significant, based on previous reports in the colorectal literature $[19,20]$.

\section{TGF $\beta$ RII mutation analysis}

The presence of somatic mutations in the poly $(\mathrm{A})_{10}$ tract of the transforming growth factor $\beta$ receptor II (TGF $\beta R I I$ ) coding sequence was investigated in MSI-H tumours by PCR followed by direct manual sequencing of the adenosine nucleotide. Both 1-bp and 2-bp deletions are predicted to result in a truncated protein product. The PCR consisted of a $50 \mu \mathrm{L}$ reaction containing 250 ng template DNA, $1.5 \mathrm{mM} \mathrm{MgCl}_{2}, 0.2 \mathrm{mM}$ nucleotides, 20 pmol forward and reverse primers, $1 \mathrm{X}$ Gold Buffer and 2U AmpliTaq Gold (Applied Biosystems). Primers were TGFBR2f: 5'-CTTTATTCTGGAAGATGCTGC-3' and TGFBR2r: 5'GATGATGTTGTCATTGCACTC-3'. Following column purification (QIAGEN) $1 \mu \mathrm{L}$ of PCR product was subjected to direct manual sequencing using the same forward and reverse primers with the Ampli-Cycle cycle sequencing kit (Perkin Elmer). The 115-bp product was electrophoresed on a $6 \%$ denaturing polyacrylamide gel for 75 minutes.

\section{Clinical correlates}

Disease-staging data was obtained for all patients according to the ACPS [21] and TNM staging systems [22]. TNM staging provided information on the extent of primary tumour invasion $(\mathrm{T})$, local lymph node spread $(\mathrm{N})$ and metastasis to a distant site (M). Patients were followed for an average of 43 months and clinical data was recorded for patient age, sex and tumour anatomical location. Tumour recurrence, death due to cancer and death due to any other cause were also noted.

\section{Statistical analysis}

Tabular data were analysed by Pearson's chi square using Web Chi Square Calculator [23] to determine significance. Non-parametric t-tests (Mann-Whitney) were performed using GraphPad Prism version 4.00 for Windows [24]. Pvalues less than 0.05 were considered statistically significant.

\section{Results}

\section{SnoN expression levels compared with MSI-status}

Transcript levels of SnoN were examined in 34 MSS and 18 MSI-H tumours relative to matched normal mucosa. There was a significantly different distribution of expression observed in MSI-H compared to MSS cancers ( $\mathrm{P}<$ 0.01 ). Whilst expression varied from 9.2 fold down-regulated to 38.1 fold up-regulated in MSI-H cancers, the majority of MSS cancers showed expression higher than matched normal mucosa levels, to a maximum of 10.5 fold (Fig. 1). Considering $>2$ fold expression differences as significant, SnoN was up-regulated in 6/18 (33\%) MSI-H cancers, whilst expression was down-regulated in $7 / 18$ $(39 \%)$ tumours in this group. By contrast, within the MSS tumour group, cancers were equally likely to demonstrate SnoN up-regulation $(17 / 34,50 \%)$ as they were to show no change in expression level $(17 / 34,50 \%)$ (Table $1, \mathrm{P}<$ $0.001)$. Significance was maintained even when the cutoff was reduced to 1.5 fold de-regulation $(\mathrm{P}<0.001)$.

\section{SnoN expression levels compared with clinical features}

No significant correlations were observed between SnoN expression levels and patient age, sex, site or ACPS stage (Table 1). Evaluation of extent of tumour invasion (T), local spread to lymph nodes $(\mathrm{N})$ or distant metastatic spread $(M)$ derived from the TNM staging system did not reveal any significant correlations. Within the MSS group, more patients without increased SnoN expression had a stage D tumour, as compared with those patients with increased SnoN expression (Table 1). Of patients with a stage $\mathrm{A}, \mathrm{B}$ or $\mathrm{C}$ tumour, 3 had a recurrence of cancer. None of these tumours showed increased SnoN expression. When these two groups were combined to indicate a poor prognosis, there was a statistically significant association between poor outcome and failure to elevate SnoN (Table $1, \mathrm{P}<0.025)$. There were only two stage D MSI-H

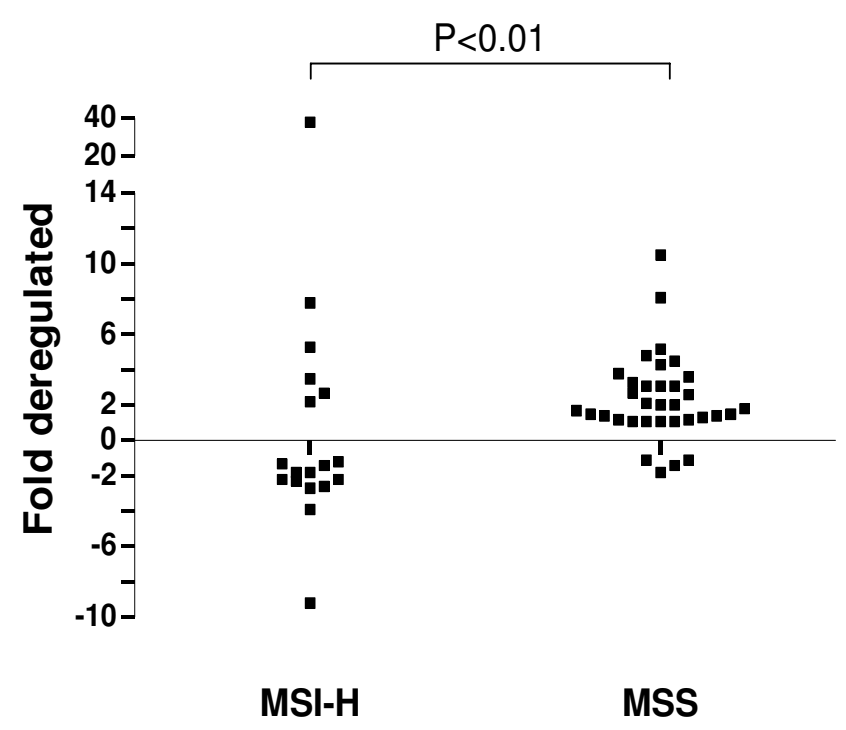

Figure I

Distribution of SnoN expression in tumours stratified by MSIstatus. SnoN data points were determined by semi-quantitative real-time PCR and are expressed as either fold up- or down-regulated, as compared to matched normal mucosa. Whilst there was a wide range of increased and decreased expression changes in the MSI-H subgroup, no MSS tumours demonstrated down-regulation greater than 2 fold. 
Table I: SnoN expression differences relative to MSI-Status and Clinical Characteristics

\begin{tabular}{|c|c|c|c|c|c|c|c|c|}
\hline & \multicolumn{4}{|c|}{ MSI-H } & \multicolumn{4}{|c|}{ MSS } \\
\hline & \multicolumn{4}{|c|}{ SnoN Expression Level } & \multicolumn{4}{|c|}{ SnoN Expression Level } \\
\hline & Total & Increased & No Change & Decreased & Total & Increased & No Change & \\
\hline Total No. Patients & 18 & 6 & 5 & 7 & 34 & 17 & 17 & $\mathrm{P}<0.00 \mathrm{I}^{\Delta}$ \\
\hline \multicolumn{9}{|l|}{ Sex } \\
\hline Male & 6 & 2 & 1 & 3 & 21 & 9 & 12 & \\
\hline Female & 12 & 4 & 4 & 4 & 13 & 8 & 5 & \\
\hline \multicolumn{9}{|l|}{ Site } \\
\hline Proximal & 13 & 3 & 5 & 5 & 14 & 8 & 6 & \\
\hline Distal & 5 & 3 & 0 & 2 & 20 & 9 & 11 & \\
\hline \multicolumn{9}{|l|}{ ACPS Stage } \\
\hline$A$ & 5 & 0 & 4 & I & 6 & 3 & 3 & \\
\hline B & 8 & 5 & 0 & 3 & 15 & 8 & 7 & \\
\hline $\mathrm{C}$ & 3 & 1 & 0 & 2 & 5 & 3 & 2 & \\
\hline $\mathrm{D}$ & 2 & 0 & 1 & 1 & 7 & 2 & 5 & \\
\hline Recurrence of Cancer in ACPS Stage A, B or C & 0 & 0 & 0 & 0 & 3 & 0 & 3 & $P<0.1 \cdot$ \\
\hline $\begin{array}{l}\text { ACPS Stage D or Recurrence of Cancer in ACPS } \\
\text { Stage A, B or C }\end{array}$ & 2 & 0 & I & 1 & 10 & 2 & 8 & $\mathrm{P}<0.025$ \\
\hline
\end{tabular}

$\triangle$ SnoN expression relative to MSI-status

- SnoN expression relative to recurrence in ACPS stage A, B or C in MSS subgroup

- SnoN expression relative to the number of patients with an ACPS stage D MSS cancer, or ACPS stage A, B or C MSS cancer which recurred

tumours, neither of which showed increased SnoN expression.

\section{TGF $\beta$ RII mutation}

Fifteen MSI-H tumours were assessed for repeat tract mutation in the coding region of the TGF $\beta R I I$ gene (Table $2)$. Mutations were detected in $12 / 15$ (80\%). Interestingly, three out of the six tumours demonstrating up-regulated SnoN expression were wild-type for this usually common mutation, whilst all nine tumours which did not have SnoN up-regulated had a mutation of either one or both TGF $\beta$ RII alleles $(\mathrm{P} \leq 0.025)$.

\section{Discussion}

SnoN is an important negative regulator of TGF $\beta$ induced tumour suppression, and exerts an oncogenic effect by binding to SMAD protein complexes [7,25]. Importantly, SnoN has also been shown to suppress tumour growth in both epithelial cell line and murine models $[9,10]$. To further delineate the role of $S n o N$ gene regulation in colorectal tumours, we quantified mRNA expression in a series of sporadic CRCs stratified by MSI-status.

Down-regulation of a gene in a significant number of tumours compared with matched normal mucosa would usually suggest abrogation of tumour suppressor gene function. Strikingly, SnoN was down-regulated in 39\% of MSI-H CRCs in this study (7/18), but not in any of the 34 MSS cancers examined $(P \leq 0.001)$. This suggests that SnoN may be an important tumour suppressor gene, exclusively in CRCs progressing via the MSI pathway of tumorigenesis. Even when the arbitrary cut-off for significant expression differences was reduced to only 1.5 fold, the statistically significant association between up-regulation of SnoN in MSS CRCs and down-regulation in MSI-H CRCs was maintained ( $\mathrm{P} \leq 0.001)$, suggesting that even subtle changes in $S n o N$ gene expression levels are capable of exerting a functional effect.

There is currently no evidence that the SnoN locus is a direct target for down-regulation. Although repeat-tract mutation and promoter hypermethylation are the major mechanisms of gene disruption in MSI-H CRCs, the SnoN transcript does not contain an extended repeat tract, nor does the 5 ' region represent a typical CpG island likely to be silenced by methylation. Whilst chromosomal instability is a characteristic feature of most MSS cancers, locus deletion is an uncommon mechanism for gene down-regulation in MSI-H tumours. It is more likely that $S n o N$ is transcriptionally down-regulated due to disruption of the negative feedback loop involving TGF $\beta$ signalling [7].

Accumulating evidence suggests that SnoN is an important regulator of TGF $\beta$ signalling, and acts predominantly through binding to TGF $\beta$ signal-transducing SMAD proteins $[7,25]$. High levels of SnoN have been shown to inhibit TGF $\beta$ signal transduction in breast [12] and skin cells [26]. In MSI-H tumours, mutation of TGF $\beta R I I$ causes resistance to TGF $\beta$-induced growth inhibition [27]. We proposed that such a situation would decrease the selec- 
Table 2: TGF $\beta$ RII mutation status relative to $S n o N$ expression

\begin{tabular}{ccccc}
\hline & \multicolumn{3}{c}{ SnoN Expression Level in MSI-H Cancers } \\
\hline & Total & Increased & No Change & Decreased \\
\hline Total No. Patients & 15 & 6 & 4 & 5 \\
TGF $\beta$ III Mutated & 12 & 3 & 4 & 5 \\
TGF $\beta$ RII Wild Type & 3 & 3 & 0 & 0 \\
\hline
\end{tabular}

tion pressure to up-regulate $S n o N$ and therefore examined the $\mathrm{A}_{10}$ repeat tract mutation of TGF $\beta R I I$ commonly observed in MSI-H cancers [28]. We found that $S n o N$ was indeed up-regulated more commonly in tumours with a wild-type receptor sequence $(P \leq 0.025)$, which may support a link between intact TGF $\beta$ signalling and $S n o N$ upregulation.

SnoN was also up-regulated compared to matched normal mucosa in a large number of MSS cancers $(17 / 34,50 \%)$, however reduced expression was not observed for any MSS tumour. This is discordant with the observed frequencies of gene amplification (15\%) and deletion (55\%) reported by Buess and colleagues [11], and highlights the importance of examining expression levels where posttranscriptional processing may further modulate transcript levels beyond the linear affect of altered copy number. Chromosomal instability is common in MSS cancers, and usually manifests as high rates of deletion of chromosomal regions where a mutated tumour suppressor gene is located on the remaining allele. It is possible that the high rate of deletion observed by Buess and colleagues is a result of another gene target on chromosome $3 q 26$, whilst expression of the remaining $S n o N$ allele is increased through compensatory transcriptional mechanisms, despite hemizygous deletion.

Luo (2004) suggested that the seemingly opposing tumour promoting and suppressing roles of SnoN may reflect the likewise dual roles of TGF $\beta$ in tumorigenesis [1]. It was proposed that early in tumour development TGF $\beta$ signalling would be activated to facilitate growth suppression. However, subsequent disruption of TGF $\beta$ signalling later in tumorigenesis could enhance the ability of TGF $\beta$ to promote tumour invasion and metastasis. Therefore, abrogation of TGF $\beta$ signalling via up-regulation of SnoN could result in tumours which are large but less likely to metastasise. This is entirely consistent with our finding of a more positive prognostic outcome in relation to cancers with increased SnoN expression. MSI is a well-established marker for a favourable prognosis [29]. This is consistent with only 2/18 of the MSI-H tumours in this series being ACPS stage D. Furthermore, it is interesting to note that neither of these cancers showed up-regulation of SnoN.
Our suggested association between failure to up-regulate SnoN and poor patient outcome in MSS cancers may be related to the role of SnoN in TGF $\beta$ signalling. This interesting observation warrants further investigation in a larger patient cohort which would allow correction for tumour stage. Comprehensive studies are now necessary to fully elucidate the connection between disrupted TGF $\beta$ signalling and the role SnoN may play in maintaining the balance between cell proliferation and differentiation.

\section{Conclusion}

SnoN transcript expression was commonly up-regulated in colorectal tumours, regardless of microsatellite instability status. Importantly, SnoN was also frequently downregulated specifically in microsatellite instability high (MSI-H) colon cancers. Based on this finding, we propose that MSI-H tumours present a unique model for examining the dual suppressive and oncogenic roles of this important regulatory gene in colorectal tumorigenesis.

\section{Competing interests}

The author(s) declare that they have no competing interests.

\section{Authors' contributions}

JC carried out the quantitative real-time PCR assays and analysis and contributed to drafting of the manuscript. LS performed the TGF $\beta$ RII mutation analysis. SC contributed to the real-time PCR assays and analysis. MW assisted with cohort selection and clinical analysis. JY contributed to study design and review of the manuscript. JJ contributed to study design and interpretation of results in the context of TGF $\beta$ signalling. KS participated in study design and review of the manuscript. BL contributed to study conception and design and collected all clinical data. VW contributed to study conception, data analysis and drafting of the manuscript. All authors read and approved the final manuscript.

\section{Acknowledgements}

This work was supported by the National Health and Medical Research Council, Queensland Cancer Fund, Queensland Health Pathology Services and the Walter Paulson Tumour Bank. 


\section{References}

I. Luo K: Ski and SnoN: negative regulators of TGF-b signalling. Current Opinion in Genetics and Development 2004, I 4:65-70.

2. Nagase T, Nomura N, Ishii S: Complex formation between proteins encoded by the ski gene family. Journal of Biological Chemistry 1993, 268:13710-13716.

3. Medrano EE: Repression of TGF-b signaling by the oncogenic protein SKI in human melanomas: consequences for proliferation, survival, and metastasis. Oncogene 2003, 22:3 I23-3 I 29.

4. Liu X, Sun Y, Weinberg RA, Lodish HF: Ski/Sno and TGF-b signalling. Cytokine Growth Factor Reviews 200 I, I 2: I-8.

5. Heyman HC, Stavnezer E: A carboxyl-terminal region of the Ski oncoprotein mediates homodimerization as well as heterodimerization with the related protein SnoN. Journal of Biological Chemistry 1994, 269:26996-27003.

6. Cohen SB, Nicol R, Stavnezer E: A domain necessary for the transforming activity of SnoN is required for specific DNA binding, transcriptional repression and interaction with TAFIII I O. Oncogene 1998, I 7:2505-25I3.

7. Stroschein SL, Wang W, Zhou S, Zhou Q, Luo K: Negative feedback regulation of TGF-b signaling by the SnoN oncoprotein. Science (Washington DC) 1999, 286:771-774.

8. Derynck R, Akhurst RJ, Balmain A: TGF-b signaling in tumour suppression and cancer progression. Nature Genetics 200I, 29: I17-129.

9. Shinagawa T, Dong H, Xu D, Maekawa T, Ishii S: The Sno gene, which encodes a component of the histone deactylase complex, acts as a tumour suppressor in mice. EMBO 2000, | 9:2280-229|

10. Sarker KP, Wilson SM, Bonni S: SnoN is a cell type-specific mediator of transforming growth factor-beta responses. The Journal of Biological Chemistry 2005, 280: | 3037-I3046.

II. Buess M, Terracciano L, Reuter J, Ballabeni P, Boulay JL, Laffer U, Metzger U, Herrmann R, Rochiltz CF: Amplification of SKI is a prognostic marker in early colorectal cancer. Neoplasia 2004, 6:207-212.

12. Zhang F, Lundin M, Ristimaki A, Heikkila P, Lundin J, Isola J, Joensuu $H$, Laiho M: Ski-related novel protein N (SnoN), a negative controller of Transforming Growth Factor-b signaling, is a prognostic marker in estrogen receptor-positive breast carcinomas. Cancer Research 2003, 63:5005-5010.

13. Fukuchi M, Nakajima M, Fukai Y, Miyazaki T, Masuda N, Sohda M, Manda R, Tsukada K, Kato H, Kuwano H: Increased expression of c-Ski as a co-repressor in transforming growth factor-b signaling correlates with progression of esophageal squamous cell carcinoma. International Journal of Cancer 2004, 1 08:8I 8-824.

14. Smith G, Carey FA, Beattie J, Wilkie MJV, Lightfoot TJ, Coxhead J, Garner RC, Steele RJC, Wolf CR: Mutations in APC, Kirsten-ras and p53 - alternative genetic pathways to colorectal cancer. Proceedings of the National Academy of Science, USA 2002, 99:9433-9438.

15. Chang SC, Lin JK, Yang SH, Wang HS, Li AF, Chi CW: Relationship between genetic alterations and prognosis in sporadic colorectal cancer. International Journal of Cancer 2006, I 1 8: I 721-1727.

16. Edmonston TB, Cuesta KH, Burkholder S, Barusevicius A, Rose D, Kovatich AJ, Boman B, Fry R, Fishel R, Palazzo JP: Colorectal carcinomas with high microsatellite instability: defining a distinct immunologic and molecular entity with respect to prognostic markers. Human Pathology 2000, 3 I: I506-I5 I4.

17. Boland CR, Thibodeau SN, Hamilton SR, Sidransky D, Eshleman JR, Burt RW, Meltzer SJ, Rodriguez-Bigas MA, Fodde R, Ranzani GN, Srivastava S: A National Cancer Institute Workshop on microsatellite instability for cancer detection and familial predisposition: Development of international criteria for the determination of microsatellite instability in colorectal cancer. Cancer Research 1998, 58:5248-5257.

18. Pfaffl MW: A new mathematical model for relative quantification in real-time RT-PCR. Nucleic Acids Research 2001, 29:2002-2007.

19. Williams NS, Gaynor RB, Scoggin S, Verma U, Gokaslan T, Simmang C. Fleming J, Tavana D, Frenkel E, Becerra C: Identification and validation of genes involved in the pathogenesis of colorectal cancer using cDNA microarrays and RNA interference. Clinical Cancer Research 2003, 9:931-946.

20. Ni HM, Leong A, Cheong D, Hooi S: Expression of CD44 variants in colorectal carcinoma quantified by real-time reverse tran- scriptase-polymerase chain reaction. Journal of Laboratory Clinical Medicine 2002, I39:59-65.

21. Newland RC, Chapuis PH, Smyth EJ: The prognostic value of substaging colorectal carcinoma. Cancer 1987, 60:852-857.

22. Wood DA: Clinical staging and end results classification: TNM system of clinical classification as applicable to carcinoma of the colon and rectum. Cancer |97|, 28:|09-|| 4 .

23. Web Chi Square Calculator [http://www.georgetown.edu/ faculty/ballc/webtools/web_chi.html].

24. GraphPad Prism version 4.00 for Windows, GraphPad Software, San Diego California USA [www.graphpad.com].

25. He J, Tegen SB, Krawitz AR, Martin GS, Luo K: The transforming activity of Ski and SnoN is dependent on their ability to repress the activity of Smad proteins. Journal of Biological Chemistry 2003, 278:30540-30547.

26. Siegel PM, Massague J: Cytostatic and apoptotic actins of TGFbeta in homeostasis and cancer. Nature Reviews 2003, 3:807-820.

27. Parsons R, Myeroff LL, Liu B, Willson JK, Markowitz SD, Kinzler KW, Vogelstein B: Microsatellite instability and mutations of the transforming growth factor beta type II receptor gene in colorectal cancer. Cancer Research 1995, 55:5548-5550.

28. Takenoshita S, Tani M, Nagashima M, Hagiwara K, Bennett WP, Yokota J. Harris CC: Mutation analysis of coding sequences of the entire transforming growth factor $\beta$ type II receptor gene in sporadic human colon cancer using genomic DNA and intron primers. Oncogene | 997, | 4: | 255- | 258.

29. Wright CM, Dent OF, Barker M, Newland RC, Chapuis H, Bokey EL, Young JP, Leggett BA, Jass JR, Macdonald GA: Prognositc significance of extensive microsatellite instability in sporadic clinicopathological stage C colorectal cancer. British Journal of Surgery 2000, 87: I 197-I202.

\section{Pre-publication history}

The pre-publication history for this paper can be accessed here:

http://www.biomedcentral.com/1471-2407/6/252/pre pub

Publish with Bio Med Central and every scientist can read your work free of charge

"BioMed Central will be the most significant development for disseminating the results of biomedical research in our lifetime. "

Sir Paul Nurse, Cancer Research UK

Your research papers will be:

- available free of charge to the entire biomedical community

- peer reviewed and published immediately upon acceptance

- cited in PubMed and archived on PubMed Central

- yours - you keep the copyright
BioMedcentral 